EESTI NSV TEADUSTE AKADEEMIA TOIMETISED.

FOUSIKA * MATEMAATIKA

ИЗВЕСТИЯ АКАДЕМИИ НАУК ЭСТОНСКОП ССР. ФИЗИКА * МАТЕМАТИКА

PROCEEDINGS OF THE ACADEMY OF SCIENCES OF THE ESTONIAN SSR.

PHYSICS * MATHEMATICS

$1985,34,2$

удК $\quad 519.3: 51: 62-50$

H. КЕНС

\title{
ЛИНЕИНОЕ НЕАВТОНОМНОЕ АГРЕГИРОВАНИЕ В СУБОПТИМАЛЬНОМ СИНТЕЗЕ УПРАВЛЕНИЙ
}

\author{
(Представил Н. Алумяэ)
}

Рассмотрено линейное неавтономное агрегирование по коордннатам многомерной линейной системы с квадратичным критерием, обобщающее стационарное агрегирование $\left[{ }^{1-5}\right]$. Получены субоптимальные регуляторы задачи и оценки модуля агрегирующей компоненты. Предложены условия точной декомпозиции системы в агрегирующих и дополнительных переменных и модификация формулы Лиувилля. Проведено исследование условия декомпозиции в его упрощенной форме (субоптимальная сепарация) как ограничения на класс допустимых матриц агрегирования в задаче поиска наилучших субоптимальных управлений по показателям вероятностного типа $\left[{ }^{3,5}\right]$.

\section{1. Постановка задачи и агрегирование исходной модели}

Рассмотрим задачу минимизации по $u$ функционала

$$
\begin{gathered}
2 f\left(t_{0}, x_{0} \mid u\right)=x_{1}^{\mathrm{T}} P_{1} x_{1}+\int_{t_{0}}^{t_{1}}\left(x^{\mathrm{T}} Q x+u^{\mathrm{T}} R u\right) d \tau, \quad \operatorname{dim} x=n \gg 1 \\
P_{1}=P\left[t_{1}\right] \geqslant 0, \quad Q[t]=Q^{\mathrm{T}}>0, \quad R[t]=R^{\mathrm{T}}>0, \\
\operatorname{dim} u=r\left(x_{\alpha}=x\left(t_{\alpha}\right), \alpha=0,1\right)
\end{gathered}
$$

в общем случае неавтономной линейной $n$-мерной системы

$$
\begin{gathered}
x=A[t] x+B[t] u ; \quad x=\left(x_{i}\right)^{\mathrm{T}} ; \quad u=\left(u_{\sigma}\right)^{\mathrm{T}}(i, s=\overline{1, n} ; \sigma=\overline{1, r}), \\
A=\left[a_{i s}(t)\right], \quad B=\left[b_{i s}(t)\right] ; \quad A, B, Q, R \subset C\left[t_{0}, t_{1}\right], \\
-\infty<t_{0}<t_{1} \leqslant \infty ; \quad t_{0}, t_{1}, x_{0}-\text { fix const. }
\end{gathered}
$$

В реальных условиях имеем два основных случая - случай неполной информации по $x$ - когда измеряется его $m$-мерная компонента

$y=\left(y_{v}\right)^{\mathrm{T}}=F(t) x, \quad F=(m \times n), \quad \operatorname{rank} F \stackrel{\Delta}{=} r_{F}=m<n, \quad t_{0} \leqslant t \leqslant t_{1}$

и случай полной информации $y=x\left(F=\mathbf{1}_{n}^{n}, v=\overline{1, m}\right)$. Случай линейно-зависимых $y\left(r_{F}<m\right)$ не имеет практического интереса. Согласно критерию Грама условие на сигнальную матрицу $r_{F}=m$ эквивалентно положительности $F F^{\mathrm{T}}>0$, когда есть обратная $\left(F F^{\mathrm{T}}\right)^{-1}$. Ниже при любой матрице $M(k \times n)(1 \leqslant k \leqslant n)$ учтем эквивалентность

$$
\operatorname{rank} M=k \sim M M^{\mathrm{T}}>0 \quad\left(\operatorname{det} M M^{\mathrm{T}}>0 \Leftrightarrow \exists\left(M M^{\mathrm{T}}\right)^{-1}\right) .
$$

Проведем в основных случаях агрегирование задачи (1.1)-(1.2) для уменьшения ее размерности $n \gg 1,2$ и, соответственно, объема памяти и вычислений на ЭВМ. В отличие от $\left[{ }^{1-6}\right]$ применим линейную нестационарную (функциональную) агрегацию с матрицами $C(t), G(t)$ вида 


$$
\begin{gathered}
z=G(t) x, \quad G=C(t) F, \quad G=(l \times n), \quad C=(l \times m) ; \quad F=(m \times n)(l \ll n) \\
G G^{\mathrm{T}}>0, \quad C C^{\mathrm{T}}>0, \quad t_{0} \leqslant t \leqslant t_{1}(1 \leqslant l \leqslant m \leqslant n)
\end{gathered}
$$

и точную линейную $z$-декомпозицию объекта $(2)$, когда для $A, G(1.2)$ существует линейная $z$-автономная при $u \equiv 0$ подсистема

$$
z=\bar{A} z+\bar{B} u=\left(G^{*}+G A\right) x+\bar{B} u\left(\bar{A}_{l}^{l}=\bar{A}(t), \bar{B}_{l}^{r}=G B\right) .
$$

Равенство (1.6) верно лишь, если $A, G, \bar{A}$ удовлетворяют условию

$$
G \cdot G A=\bar{A} G \quad(G(t)=(l \times n), \quad A=A(t)),
$$

что эквивалентно ввиду (1.5) равенству (1.8) и $l(n-l)$-независимым условиям на $A, G$ вида

$$
\begin{aligned}
& \bar{A}=\left(G^{\cdot}+G A\right) G^{\mathrm{T}}\left(G G^{\mathrm{T}}\right)^{-1}, \\
& \widetilde{P}_{0} \widetilde{Q}_{0}=(G+G A)\left[G^{\mathrm{T}}\left(G G^{\mathrm{T}}\right)^{-1} G-1_{n}^{n}\right]={ }_{l} 0^{n}, \\
& \widetilde{P}_{0} \triangleq\left(G^{\cdot}+G A\right), \quad{ }_{0} \triangleq\left[G^{\mathrm{T}}\left(G G^{\mathrm{T}}\right)^{-1} G-1_{n}^{n}\right],
\end{aligned}
$$

где $\varnothing_{0}-$ симметричная неположительная матрица ранга $n-l$ c $(n-l)$ кратным собственным $(-1)$ и $l$-кратным нулевым числом, которому отвечает $l$-мерное собственное подпространство с базисом из $\mathrm{col} G^{\mathrm{T}}$. Поэтому условие (1.9) эквивалентно равенству с произвольной $(l \times l)$ мерной матрицей $W(t, \cdot)$,

где $G \cdot+G A=W G$.

Из (1.6),(1.8), (1.10) находим, что z-подсистема и (1.7) должны удовлетворять

$$
z=\bar{A} z+\bar{B} u, \quad G \cdot=\bar{A} G-G A \quad(\bar{A} \equiv W),
$$

где достаточно считать $\bar{A}$ независящей от $G$, полагая для линейности по $G$, что $\bar{A}=\bar{A}(t)$. Произвол выбора $\bar{A}(t)$ используется 'в оптимизации приближенного решения исходной задачи. Матрицант $G(t, \cdot)$, заданный (1.11), имеет вид

$G(t, \tau)=\Phi \frac{A}{-} G(\tau, \tau) \Phi_{A}^{-1}(t, \tau), \quad r_{G}=r_{G \tau}=l, \quad G_{\tau} \triangleq G(\tau, \tau), \quad \Phi-(t)=(l \times l)$,

где $\Phi_{\bar{A}}(t), \Phi_{A}(t)-$ переходные матрицы, отвечающие уравнениям

$$
\Phi_{\bar{A}}^{\cdot}=\bar{A} \Phi_{\bar{A}}, \quad \Phi_{\bar{A}}(\tau ; \tau)=1_{l}^{l} ; \Phi_{A}^{\cdot}=A \Phi_{A}, \Phi_{A}(\tau, \tau)=1_{n}^{n} \quad\left(t_{0} \leqslant \tau \leqslant t_{1}\right) .
$$

Легко показать, что $r[G(t, \cdot)]=l$, если $r G_{\tau}=l$. Это следует также из $(1.12)$ и модификации формулы Лиувилля при $l \leqslant n$. С целью ее вывода рассмотрим аналогичное (1.11) уравнение

$$
\hat{G}^{\cdot}=\hat{A} \hat{G}+\hat{G} \hat{B} \quad(\hat{G}=\hat{G}(t, \cdot)=(l \times n), l \leqslant n) .
$$

Введем матрицу $M \stackrel{\Delta}{=} \hat{G} \hat{G}^{\mathrm{T}}$,-определенную уравнением

$$
M \cdot=\hat{A} M+M \hat{A}+\hat{G} P \hat{G}^{\mathrm{T}}, \hat{P} \stackrel{\Delta}{=} \hat{B}+\hat{B}^{\mathrm{T}}, \quad M \stackrel{\Delta}{=} \Phi_{\hat{A}}^{\mathrm{T}} N \Phi_{\hat{A}}^{\mathrm{T}} \quad\left(M=M^{\mathrm{T}}\right),
$$

$N \cdot=S \hat{P} S, N=S S^{\mathrm{T}}, S=\hat{G}_{\tau} \Psi_{\hat{B}}=(l \times n), N(\tau, \cdot)=\left.S S^{\mathrm{T}}\right|_{t=\tau}=(l \times l) ;(1.13)$ $\Phi_{\hat{A}}=\hat{A} \Phi \hat{\hat{A}}, \Phi_{\hat{A}}(\tau, \tau)=1_{l}^{l} ; \quad \Psi_{\hat{B}} \stackrel{\Delta}{=} \Psi_{\hat{B}} \hat{B}, \Psi_{\hat{B}}(\tau, \tau)=1_{n}^{n}\left(t_{1} \leqslant \tau \leqslant t_{2}\right)$. 
Так как $r_{\mathrm{S}}=l$, то из $(1.4),(1.13)$ имеем $N^{-1}$ и равенства

$$
\begin{aligned}
& N \cdot=\left[S \hat{P} S^{\mathrm{T}} N^{-1}\right] N \quad(\Delta X \triangleq \operatorname{det} X), \\
& (\Delta N)=\operatorname{tr}\left[S \hat{P} S^{\mathrm{T}} N^{-1}\right] \Delta N,
\end{aligned}
$$

в которых найдем след матрицы $S \hat{P} S^{\mathrm{T}} N^{-1}$, учитывая, что следы $X, \hat{T} X \hat{T}^{-1}$ одинаковые при $\Delta \hat{T} \neq 0$. Так как $N>0$ и $N$ - симметрические, то имеем матрицу $T(l \times l), r(T)=l, \Delta T \neq 0$, такую, что

$$
\begin{gathered}
S \hat{P} S^{\mathrm{T}}=T^{*-1} \Lambda T^{-1} ; \quad N=S S^{\mathrm{T}}=T^{*-1} T^{-1}, \quad \Lambda=\operatorname{diag}\left(\beta_{1}, \ldots, \beta_{l}\right), \\
\Lambda=T^{*} S \hat{P} S^{\mathrm{T}} T=Q^{*} \hat{P} Q, \quad Q \stackrel{\Delta}{=} S^{\mathrm{T} T} \quad\left(Q^{*} \stackrel{\Delta}{=} Q^{\mathrm{T}}, T^{*} \stackrel{\Delta}{=} T^{\mathrm{T}}\right), \\
\chi(\beta) \stackrel{\Delta}{=}\left|S \hat{P} S^{\mathrm{T}}-\beta N\right|=0 \Rightarrow \beta_{\lambda}, \quad \lambda=\overline{1, l ;} \quad S \hat{P} S^{\mathrm{T}} N^{-1}=T^{*-1} \Lambda T^{*} .
\end{gathered}
$$

Согласно (1.15) матрица $Q(n \times l)-$ обобщенно-ортогональная $Q^{*} Q=$ $=\mathbf{1}_{l}^{l}$ вида

$$
Q^{*}=\left[Q_{1}^{*} \mid 0_{l}^{n-l}\right], \quad Q_{1}^{*} Q_{1}=\mathbf{1}_{l}^{l}, \quad Q_{1}=(l \times l), \quad Q_{1}^{\mathrm{T}} \stackrel{\Delta}{=} Q^{*} .
$$

Из (1.15), (1.16) находим равенства

$$
\begin{gathered}
\operatorname{tr} S \hat{P} S^{\mathrm{T}} N^{-1}=\operatorname{tr} \Lambda=\operatorname{tr} Q_{1}^{*} P_{11}^{*} Q_{1}=\operatorname{tr} \hat{P}_{11}=2 \operatorname{tr} \hat{B}_{11}, \\
\hat{P}=\left[\begin{array}{ll}
\hat{P}_{11} & \hat{P}_{12} \\
\hat{P}_{21} & \hat{P}_{22}
\end{array}\right], \quad \hat{P}_{11}=(l \times l) ; \quad \hat{B}=\left[\begin{array}{cc}
\hat{B}_{11}, & \hat{B}_{12} \\
\hat{B}_{21}, & \hat{B}_{22}
\end{array}\right], \quad \hat{B}_{11}=(l \times l),
\end{gathered}
$$

в силу которых (1.14) принимает вид $(\Delta N)^{\circ}=2 \operatorname{tr} B_{11}(\Delta N)$. С учетом (1.13) из (1.17) находим для $G$ модификацию формулы Лиувилля

$$
\Delta M=\Delta M(\tau) \exp 2 \int_{\tau}^{t} \operatorname{tr}\left[\hat{A}(\sigma)+\hat{B}_{11}(\sigma)\right] d \sigma \quad\left(M \stackrel{\Delta}{=} \hat{G} \hat{G}^{\mathrm{T}}\right)
$$

при $\hat{G}=\hat{A} \hat{G}+\hat{G} \hat{B}, \hat{G}=(l \times n), \quad \hat{A}=(l \times l), \quad \hat{B}=(n \times n), \quad 1 \leqslant l \leqslant n$, доказывающую, что $r \hat{G}(t, \tau)=r\left(G_{\tau}\right)=l$. Из (1.17) следует также, что сумма корней $\beta_{i}$ характернстического уравнения в $(1.15)$ равна удвоенному следу субматрицы $\hat{B}_{11}(l \times l)$ в $\hat{B}$ при любом выборе $S$ ранга $l$. Собственные числа субматрицы $\hat{B}_{11}+\hat{B}_{11}^{\mathrm{T}}$ совпадают с корнями $\chi(\beta)=0$ при $r(S)=l$. В случае $(1.11)$ из (1.18) имеем

$$
\Delta \cdot\left[G G^{\mathrm{T}}\right]=2 \Delta\left[G G^{\mathrm{T}}\right] \operatorname{tr}\left[\bar{A}-A_{11}\right], \quad A_{11}=(l \times l), \quad A=\left[\begin{array}{ll}
A_{11} & A_{12} \\
A_{21} & A_{22}
\end{array}\right] .
$$

При $G$-линейности (1.11) в случае точной $z$-линейной декомпозиции (1.2) необходимо и достаточно задать матрицу декомпозиции $G$ согласно (1.12) при произвольной постоянной $G_{\tau}$ ранга $l$. Альтернативные случаи (1.3) неразличимы в (1.11) и ниже рассматриваются вместе. Их различие - в смысле матриц агрегации $C$ и декомпозиции $G$. При измерении всех $x_{i}$ они совпадают $C=G$, а класс допустимых $C(l \times n)$ находим из $(1.12)$ заменой $G \rightarrow C, G_{\tau} \rightarrow C_{\tau}, r(C)=l$. В сигнальном случае $(C F=G, m<n)$ ввиду (1.12) матрицы $C, F$ связаны конфликтным по свободе их выбора условием 


$$
\hat{C} \hat{F}=G_{\tau}=\text { const } ; \quad C=\Phi_{A}^{-1} C, \quad \hat{F}=F(t) \Phi_{A}(t, \tau) .
$$

Для выполнения (1.20) необходимо и достаточно, чтобы

$$
\hat{C}=G_{\tau} \hat{F}^{\mathrm{T}}\left(\hat{F} \hat{F}^{\mathrm{T}}\right)^{-1}, \quad G_{\tau} \hat{Q}={ }_{l} 0^{n} \quad\left(\hat{Q} \stackrel{\Delta}{=} \mathbf{1}_{n}^{n}-\hat{F}^{\mathrm{T}}\left(\hat{F} \hat{F}^{\mathrm{T}}\right)^{-1} \hat{F}\right)
$$

Второе из (1.21) верно лишь при $G_{\tau}=S \hat{F}$ с произвольной $S(l \times n)$. Последнее, в частности, означает, что строки взятой $G_{\tau}$ - линейные комбинации строк $F$. Аналогично (1.8), (1.9), собственные числа $\hat{Q}$ суть $m$-кратный нуль и $(n-m)$-кратная единица. Используя субматрицы $S^{0}, S^{1}$ диагонализирующей $\hat{Q}$ ортогональной $S(n \times n)$-матрицы

$$
S=\left[S^{0} \mid S^{1}\right], \quad S^{0}=\hat{F}^{\mathrm{T}} \hat{N}=(n \times m), \quad \hat{N} \hat{N}^{\mathrm{T}}=\left(\hat{F} \hat{F}^{\mathrm{T}}\right)^{-1},
$$

сводим второе равенство (1.21) к системе $l \times(n-m)$ независимых равенств

$$
\begin{gathered}
G_{\tau} S^{1}={ }_{l} 0^{N}, \hat{F} S^{1}={ }_{m} 0^{N}, \quad S^{1}=\left[s^{m+1}, \ldots, s^{n}\right]=(n \times N), \\
r\left(S^{1}\right)=N \triangleq n-m, \quad S^{\mu}=\left[s_{i \mu}\right], \quad \mu=\overline{m+1, n}, \quad i=\overline{1, n} ; \quad S^{0 \mathrm{~T}} S^{1}={ }_{m} 0^{N} .
\end{gathered}
$$

Максимально освобождая $\hat{F}(m \times n)(1.20)$, из (1.21) получим

$$
C=\Phi_{A} \tilde{C}=\Phi_{-} G_{\tau} \hat{F}^{\mathrm{T}}\left(\hat{F} \hat{F}^{\mathrm{T}}\right)^{-1} \quad\left(\tilde{C}=\Phi_{\bar{A}}^{-1} C, \hat{F}=F \Phi_{A}\right)
$$

Для выполнения (1.22) необходимо и достаточно, чтобы сигнальные матрицы $F(t)$ составляли допустимый класс, определенный равенствами

$F=\widetilde{P}\left[\begin{array}{c}G_{\tau} \\ \tilde{H}\end{array}\right] \Phi_{A}^{-1}, r\left[\begin{array}{c}G_{\tau} \\ \tilde{q}\end{array}\right]=m, \Delta \widetilde{P} \neq 0 ; \widetilde{P}(m \times m), \tilde{H}_{m-l}^{n}-$ произвольны

Вид допустимых матриц агрегации $C$ находим подстановкой (1.24) в (1.23). Всякая $F$-матрица, в частности, $\hat{F}, F(t)$ в (1.24), сводится нормирующей матрицей $N^{\mathrm{T}}(m \times m), N N^{\mathrm{T}}=(F F)^{-1}$ к обобщенно-ортогональной $F^{*}=N^{\mathrm{T}} F$, где $F^{*} F^{* \mathrm{~T}}=\mathbf{1}_{m}{ }^{m}$. Используя вместо $y$ вектор $\hat{y}=\hat{N}^{\mathrm{T}} y$, заменим $\hat{F}$ в $(1.20)-(1.24)$ на $\hat{F}^{*}=\hat{N}^{\mathrm{T}} \hat{F}$. Тогда класс допустимых $C$ согласно $(1.23),(1.24)$ и $\hat{F} \rightarrow \hat{F}^{*}$ имеет вид

$$
C=C^{*}=\Phi_{-} G_{\tau} \hat{F}^{*} \quad \text { при } \quad \hat{F} \rightarrow \hat{F}^{*}, \quad \hat{F}^{*} \hat{F}^{* \mathrm{~T}}=\mathbf{1}_{m}^{m} \Leftrightarrow \hat{N} \hat{N}^{\mathrm{T}}=\left(\hat{F} \hat{F}^{\mathrm{T}}\right)^{-1} .
$$

При условии свободы выбора $C$, достигаемой $(l \times m)$-дополнительными ограничениями на $F$, надо различать подсигнальную $(l<m)$ - и эквисигнальную $(l=m)$-агрегацию. С учетом $(1.20)$ и произвола $C$ из

$$
C F=G=\Phi_{\bar{A}} G_{\tau} \Phi_{A}^{-1} \quad\left(\Phi_{\bar{A}}=\Phi_{\bar{A}}(t, \tau), \Phi_{A}=\Phi_{A}(t, \tau) ; G_{\tau}=G(\tau, \tau)\right)
$$

имеем при любых $C, r(C)=l$ совокупность допустимых $F(t)$-матриц

$$
F=C^{\mathrm{T}}\left(C C^{\mathrm{T}}\right)^{-1} G+D F_{0}, \quad F_{0}=(d \times n)-\text { произвольная } \tilde{F}_{0}(t)
$$

$$
D: C D={ }_{l} 0^{d}, \quad r(D)=d \stackrel{\Delta}{=} m-l, \quad D=(m \times d) .
$$

Значения (1.26) обращают (1.25) в тождество и необходимы. В экви- 
сигнальном случае решение $C$-однородной системы тривиально в отличие от подсигнального случая, где $D F_{0} \neq 0$. При $F$, заданных $(1.26)$, нет никаких дополнительных условий на допустимые $C, G$.

\section{2. Агрегирование по функционалу}

Примем упрощение $P=\alpha^{2}(t) Q$. Введем дополняющую $z$-компоненту $y$ из координат $y_{v}, y=\left(y_{v}\right)^{\mathrm{T}}, z=\left(z_{\lambda}\right)^{\mathrm{T}}, v=\overline{1, N} ; \lambda=\overline{1 ; l}$ вида

$$
y=H x, \quad r(H)=N \stackrel{\Delta}{=} n-l, \quad H=(N \times n), \quad T \stackrel{\Delta}{=}\left[\begin{array}{l}
G \\
H
\end{array}\right], \quad \Delta T \neq 0,
$$

где $\operatorname{col} G^{\mathrm{T}}, H^{\mathrm{T}}$ удовлетворяют на $\mathfrak{I} \triangleq\left[t_{0}, t_{1}\right]$ условию $Q^{-1}$-ортогональности

$$
G Q^{-1} H^{\mathrm{T}}={ }_{l} 0^{N} \sim Q=G^{\mathrm{T}}\left[G Q^{-1} G^{\mathrm{T}}\right]^{-1} G+H^{\mathrm{T}}\left[H Q^{-1} H^{\mathrm{T}}\right]^{-1} H,
$$

эквивалентному разделению по $z, y$ в $\xi=T x$ квадрик $x^{\mathrm{T}} Q x, x^{\mathrm{T}} P x$ :

$$
\begin{gathered}
x^{\mathrm{T}} Q x=\xi^{\mathrm{T}} \widetilde{Q}=z^{\mathrm{T}} \widetilde{Q}_{1} z+y^{\mathrm{T}} \widetilde{Q}_{2} y, \quad x_{1}^{\mathrm{T}} P_{1} x_{1}=\xi_{1}^{\mathrm{T}} \widetilde{P}_{1} \xi_{1}=\alpha_{1}^{2}\left(z_{1}^{\mathrm{T}} \widetilde{Q}_{11} z_{1}+\dot{y}_{1}^{\mathrm{T}} \widetilde{Q}_{21} y_{1}, \quad, \quad(2.3)\right. \\
S=T^{\mathrm{T}} \tilde{S} T, \quad \Psi(x) \stackrel{\Delta}{=} \tilde{\Psi}(\xi)=\tilde{\Psi}(T x), \quad \widetilde{Q}_{\gamma_{1}} \stackrel{\Delta}{=} \widetilde{Q}_{\nu}\left[t_{1}\right], \quad \xi_{1} \stackrel{\Delta}{=} \xi\left[t_{1}\right], \quad \gamma=1,2, \\
\widetilde{Q}_{1} \stackrel{\Delta}{=}\left(G Q^{-1} G^{\mathrm{T}}\right)^{-1}>0, \quad r\left(\widetilde{Q}_{1}\right)=l ; \quad \widetilde{Q}_{2}=\left(H Q^{-1} H^{\mathrm{T}}\right)^{-1}, \quad r\left(\widetilde{Q}_{2}\right)=N .
\end{gathered}
$$

В силу (2.1) - (2.3) функционал (1.1) принимает вид

$$
f=\tilde{f}=\tilde{f}_{1}+\tilde{f}_{2} ; \quad 2 \tilde{f}_{1}=z_{1}^{\mathrm{T}} \widetilde{P}_{11} z_{1}+\int_{t_{0}}^{t_{1}}\left(z^{\mathrm{T}} \widetilde{Q}_{1} z+u^{\mathrm{T}} R u\right) d \tau,
$$

$$
2 \tilde{f}_{2}=y_{1}^{\mathrm{T}} \tilde{P}_{21} y_{1}+\int_{t_{0}}^{t_{1}} y^{\mathrm{T}} \widetilde{Q}_{2} y d \tau \quad\left(\widetilde{P}_{\gamma_{1}}=\alpha_{1}^{2} Q_{\gamma 1}, \alpha_{1}^{2}=\alpha^{2}\left[t_{1}\right], \alpha^{2} \geqslant 0\right) .
$$

С учетом (1.2), (1.11), (2.1) $z[t], y[t]-$ компоненты $\xi$ удовлетворяют системе

$$
\begin{gathered}
z=\bar{A} z+\bar{B} u, \quad y=\hat{F} z+\overline{\bar{A}} y+\overline{\bar{B}} u \quad(\bar{B}=G B, \bar{B}=H B), \\
\hat{F}=(H \cdot H A) T_{1}^{-1}, \quad \bar{A}=(H \cdot H A) T_{2}^{-1},-T^{-1}=\left[T_{1}^{-1} \mid T_{2}^{-1}\right], T_{1}^{-1}=(n \times l), \\
G \cdot=\bar{A} G-G A, \quad G=\Phi_{\bar{A}} G_{1} \Phi_{A}^{-1} ; \quad G_{1}=G\left[t_{1}, \cdot\right], \quad r(G)=l, \quad T T-1 \stackrel{\Delta}{=} 1_{n}^{n}, \\
\Phi_{C}: \Phi_{C}^{\cdot}=C \Phi_{C}, \Phi(\tau, \tau, \cdot)=1_{k}^{k}, \quad \forall C=(k \times k)\left(\Phi\left(t_{1}, t_{3}\right)=\Phi\left(t_{1}, t_{2}\right) \Phi\left(t_{2}, t_{3}\right)\right) .
\end{gathered}
$$

Согласно (2.4) введем в переменных $\xi$ агрегат функционала (1.1)

$2 \bar{f}=z_{1}^{\mathrm{T}} \widetilde{P}_{11} z_{1}+\int_{t_{0}}^{t_{1}}\left(z^{\mathrm{T}} \bar{Q}_{1} z+u^{\mathrm{T}} R u\right) d \tau \rightarrow \min _{u} 2 \bar{f} \stackrel{\Delta}{=} \bar{S}=2 \bar{f}\left(\cdot \mid u^{*}\right), \quad u^{*} \triangleq \underset{u}{\operatorname{argmin}} \bar{f}$,

где $\bar{Q}_{1}=\widetilde{Q}_{1}+\Gamma$. $\Gamma^{\mathrm{T}}=\Gamma-$ компенсационную матрицу невязки найдем ниже. Трактуя $l$-мерную $z$-автономную подсистему (2.5) с критерием (2.6) как агрегат системы (1.1), (1.2), получим ее оптимальный регулятор $u^{*}$ и оптимум $\bar{S}$ агрегации 
$u^{*}=-R^{-1} \bar{B}^{\mathrm{T}} \bar{M} z ; \quad \bar{S}=\bar{f}\left(\cdot \mid u^{*}\right) \triangleq \bar{f}^{*}=1 / 2 z^{\mathrm{T}} \bar{M} z \quad\left(\bar{M}^{\mathrm{T}}=\bar{M}(t)=(l \times l)\right)$,

где матрицант $\bar{M}(t, \cdot)$ - краевое решение матричного уравнения

$$
\bar{M} \cdot=\bar{M} \bar{B} R^{-1} \bar{B}^{\mathrm{T}} \bar{M}-\left(\bar{A}^{\mathrm{T}} \bar{M}+\bar{M} \bar{A}\right)-\bar{Q}, \quad \bar{M}\left(t_{1}, t_{1}, \cdot\right)=\alpha_{1}^{2} \widetilde{Q}_{11} .
$$

Замыкая (2.4), (2.5), эквивалентные (1.1), (1.2), ее субоптимальным регулятором (2.7), получим модель и квадрику субоптимума (2.4)

$$
\begin{gathered}
z=\bar{A}^{*} z, \quad y=\overline{\bar{A}} y+\hat{F}^{*} z, \quad \widehat{S}^{*} \triangleq \tilde{f}\left(\cdot, u^{*}\right)=1 / 2 \xi^{\mathrm{T}} \tilde{L} \xi \\
L=\left[\begin{array}{cc}
\bar{M} & \widetilde{R} \\
\widetilde{R}^{\mathrm{T}} & \widetilde{K}
\end{array}\right], \quad \bar{A}^{*}=\bar{A}-\overline{\bar{B}} R^{-1} \bar{B}^{\mathrm{T}} \bar{M}, \quad \hat{F}^{*}=\hat{F}-\bar{B} R^{-1} \bar{B}^{\mathrm{T}} \bar{M}, \\
(\widetilde{M}(l \times l), \widetilde{R}(l \times N) ; \widetilde{K}(N \times N)),
\end{gathered}
$$

где $\widetilde{K}, \widetilde{R}, \widetilde{M}$ - решения последовательно связанных линейных уравнений

$$
\begin{gathered}
-\widetilde{K}=\overline{\bar{A}} \widetilde{K}+\widetilde{K} \overline{\bar{A}}+\widetilde{Q}_{2}, \widetilde{K}_{1}=\alpha_{1}^{2} Q_{21} \quad\left(\widetilde{Q}_{2}=\left(H Q^{-1} H^{\mathrm{T}}\right)^{-1}, \bar{Q}_{\gamma_{1}}=\widetilde{Q}_{\nu}\left[t_{1}\right]\right), \\
-\widetilde{R}=\hat{F}^{* \mathrm{~T}} \widetilde{K}+\bar{A}^{* \mathrm{~T}} \widetilde{R}+\widetilde{R} \overline{\bar{A}}, \quad \widetilde{R}_{1}=0 \\
\left(\widetilde{Q}_{1}=\left(G Q^{-1} G^{\mathrm{T}}\right)^{-1}, \widetilde{R}_{1}=\widetilde{R}\left[t_{1}\right], \quad \widetilde{K}_{1}=\widetilde{K}\left[t_{1}\right]\right), \\
-\widetilde{M} \cdot=\widetilde{Q}_{1}+\widetilde{M} \bar{A}^{*}+\bar{A}^{* \mathrm{~T}} \widetilde{M}+\widetilde{R} \hat{F}^{*}+\hat{F}^{* \mathrm{~T}} \widetilde{R}^{\mathrm{T}}+\bar{M}^{\mathrm{T}} \bar{B} R^{-1} \bar{B}^{\mathrm{T}} \bar{M}, \quad \widetilde{M}_{1}=\alpha_{1}^{2} \widetilde{Q}_{11} .
\end{gathered}
$$

Матрица $\Gamma$ дана условием $\bar{M} \equiv \bar{M}$ эквивалентным из $(2.8)-(2.10)$ равенству

$$
\Gamma=\widetilde{R} \hat{F}^{*}+\hat{F}^{* \mathrm{~T}} \widetilde{R}^{\mathrm{T}} \quad\left(\Gamma^{\mathrm{T}} \equiv \Gamma\right) .
$$

Из уравнения на $\widetilde{R}$ следует, что $\widetilde{R} \equiv 0$ лишь при $\hat{F}^{*}=\hat{F}-\overline{\bar{B}} R^{-1} \bar{B}^{\mathrm{T}} \bar{M}=0$. Тогда в силу $(2.6)-(2.11)$ получаем упрощения

$$
\Gamma \equiv 0, \quad \bar{Q}_{1} \equiv \widetilde{Q}_{1} ; \quad \bar{M}=\widetilde{M} \quad \text { при } \quad \hat{F}=\hat{F} \triangleq \triangleq \bar{B} R^{-1} \bar{B}^{\mathrm{T}} M .
$$

Согласно $(2.1),(2.5)$ матрицы $H, \overline{\bar{A}}, \hat{F}$ удовлетворяют связи

$$
H \cdot H A-\overline{\bar{A}} H=\hat{F} G \text {. }
$$

При произвольной $\overline{\bar{A}}$ и данной $G$ из совместности $(2.13)$ для $H$ необходимо

$$
(H+H A-\overline{\bar{A}} H)\left[G^{\mathrm{T}}\left(G G^{\mathrm{T}}\right)^{-1} G-\mathbf{1}_{n}^{n}\right]={ }_{N} 0^{n} \quad(N=n-l) .
$$

Аналогично (1.7)-(1.11) ограничимся в (2.13), (2.14) при $\hat{F}=\hat{F}_{*}(t)$ случаем линейной по $H$ связи

$$
H \cdot=\overline{\bar{A}} H-H \Omega, \quad H=\Phi_{\bar{A}} H_{1} \Phi_{\Omega}^{-1} ; \Omega \triangleq A-\omega, \omega \triangleq B R^{-1} \bar{B}^{\mathrm{T}} \bar{M} G
$$

Обратно, если определить $H=H(t)$ уравнением (2.15), то при $u=u^{*}$ выполняются тождества $(2.12), \quad \widetilde{R} \equiv 0, \hat{F} \equiv \hat{F}_{*}$, а подсистемы на $z(t)$, $y(t), \widetilde{M}(t), \widetilde{K}(t)$ в $(2.9),(2.10)$ разделяются 


$$
\begin{gathered}
z=\bar{A}^{*} z, \quad y=\overline{\bar{A}} y \quad\left(G=\Phi_{\bar{A}} G_{1} \Phi_{A}^{-1}, H=\Phi_{A}^{-} H_{1} \Phi_{\Omega}^{-1}, H_{1}=H\left(t_{1}, \cdot\right)\right), \\
-\widetilde{M}=\bar{A}^{* \mathrm{~T}} \widetilde{M}+\widetilde{M} \bar{A}^{*}+\widetilde{Q}_{1}+\bar{M}^{\mathrm{T}} \bar{B} R^{-1} \bar{B}^{\mathrm{T}} \bar{M}, \quad \widetilde{M}_{1}=\alpha_{1}^{2} \widetilde{Q}_{11} \quad(\widetilde{M}=\bar{M}), \\
-\widetilde{K} \cdot=\overline{\bar{A}} \widetilde{K}+\widetilde{K} \bar{A}+\widetilde{Q}_{2}, \quad \widetilde{K}_{1}=\alpha_{1}^{2} \widetilde{Q}_{21} ; \quad \bar{A}(N \times N)-\text { произвольная } \overline{\bar{A}}(t) .
\end{gathered}
$$

С учетом равенств и обозначений (2.4), (2.9) функционал (1.1) на $u^{*}$ в $t$, छ при геометрическом условии (2.2) имеет вид

$$
2 \tilde{f}^{*}\left(u^{*} \mid t, \xi\right) \triangleq 2 \tilde{S}^{*}(t, \xi)=z^{\mathrm{T}} \bar{M} z+y^{\mathrm{T}} \widetilde{K} y \quad\left(G Q^{-1} H^{\mathrm{T}}={ }_{l} 0^{N}\right) .
$$

\section{Оценки модуля $z(t)$-компоненты $x(t)$ на $\mathfrak{I}$}

Рассмотрим оптимум агрегации $\bar{S}$ и $\bar{S}$. в силу $(2.16)$

$$
\bar{S}=1 / 2 z^{\mathrm{T}} \bar{M} z, \quad-\bar{S}^{\cdot}=1 / 2 z^{\mathrm{T}} \hat{Q}_{1} z \quad\left(\hat{Q}_{1}=\widetilde{Q}_{1}+\bar{M} G B R^{-1} B^{\mathrm{T}} G^{\mathrm{T}} \bar{M}>0\right) .
$$

Существует $z=V[t] v$ преобразование, приводящее (2.18) к виду

$$
\begin{gathered}
2 S=\sum_{\lambda=1}^{l} v_{\lambda}^{2},-2 S=\sum_{\lambda=1}^{l} k_{\lambda}^{2} v_{\lambda}^{2}, \quad-k_{l}^{2}(t) S \leqslant S \leqslant-k_{1}^{2}(t) S(S(v)=S(z)), \\
V^{\mathrm{T}} \bar{M} V=1_{l}^{l}, \quad V^{\mathrm{T}} \hat{Q}_{1} V=\operatorname{diag} k_{\lambda}^{2}, \\
\chi\left(k^{2}\right) \triangleq\left|\hat{Q}_{1}-k^{2} \bar{M}\right|=0<k_{1}^{2}=\min k^{2} \leqslant k_{l}^{2}=\max k^{2} .
\end{gathered}
$$

Из (2.18) и (2.19) получаем неравенства

$$
\begin{gathered}
2 S / m_{l}^{2} \leqslant\|z\|^{2} \leqslant 2 S / m_{1}^{2}, \quad \xi\left(m^{2}\right) \triangleq\left|\bar{M}-m^{2} \mathbf{1}_{l}^{l}\right|=0<m_{1}^{2}=\min m^{2} \\
\left(t_{0} \leqslant t \leqslant t_{1}\right), \\
S_{0} \exp \left[-\int_{t_{0}}^{t} k_{l}^{2}(\tau) d \tau\right] \leqslant S \leqslant S_{0} \exp \left[-\int_{t_{0}}^{t} k_{1}^{2}(\tau) d \tau\right] \quad\left(m_{l}^{2}=\max m^{2}\right), \\
m_{10}^{2} \leqslant 2 S_{0}\left\|z_{0}\right\|^{-2} \leqslant m_{l 0}^{2} \quad\left(m_{\alpha 0} \triangleq m_{\alpha}\left(t_{0}\right), m_{\alpha}=m_{\alpha}(t), S_{0}=\bar{S}\left(t_{0}\right), \alpha=1 ; l\right) .
\end{gathered}
$$

Согласно $(2.19),(2.20)$ норма $z(t)$-компоненты удовлетворяет оценкам на $\mathfrak{I}$ вида

$$
m_{10}^{2} m_{l}^{-2} \exp \int_{t}^{t_{0}} k_{l}^{2} d \tau \leqslant\left\|z_{0}\right\|^{-2}\|z\|^{2} \leqslant m_{l 0}^{2} m_{0}^{-2}(t) \exp \int_{t}^{t_{0}} k_{1}^{2} d \tau,
$$

где на $\tau_{j} \in \mathfrak{I}$ возможен численный поиск $m_{\lambda}{ }^{2}\left(\tau_{j}\right), k^{2}\left(\tau_{j}\right)$ ввиду малости $l \ll n\left(j=\overline{0, p}<\infty, \tau_{p}=t_{1}\right.$ при $\left.t_{1}<+\infty\right)$.

\section{3. Условие декомпозиции и его упрощение (субоптимальная сепарация)}

Рассмотрим ограничения на $(1.2)$ и матрицы $\bar{A}, G_{1}$ согласно (2.2). В силу $(1.25),(2.15)$ это условие эквивалентно

$$
G_{1} \Phi_{A}^{-1} Q^{-1} \Phi_{\Omega}^{-1} H_{1}^{\mathrm{T}}={ }_{l} 0^{N} \quad\left(\text { при } t=t_{1} \text { имеем } G_{1} Q_{1}^{-1} H_{1}^{\mathrm{T}}={ }_{l} 0^{N}\right),
$$


$H_{1}^{\mathrm{T}}=Q_{1} P_{1} W_{1}^{0}, \quad P_{1}=P_{1}\left(G_{1}\right): G_{1} P_{1}=10^{N}, \quad P_{1}^{\mathrm{T}} P_{1}=\mathbf{1}_{N}^{N}, \quad W^{0}(N \times N)=W^{0}[t]$,

$$
G_{1}^{\mathrm{T}}\left(G_{1} G_{1}^{\mathrm{T}}\right)^{-1} G_{1}+P_{1} P_{1}^{\mathrm{T}}=\mathbf{1}_{n}^{n}, \quad P_{1}=\mathrm{const}, \quad \Delta W^{0}[t] \neq 0, \quad W_{1}^{0}=W^{0}\left[t_{1}\right],
$$

где $W^{0}[t]-$ произвольная $(N \times N)$-матрица на $\mathfrak{I}$.

Введем $U(l \times l), V(N \times N)$ - произвольные матрицанты $t$ ранга $l, N$ и обозначим

$\bar{G}_{1} \triangleq\left(G_{1} G_{1}^{\mathrm{T}}\right)^{-1 / 2} G_{1}, \quad N^{\mathrm{T}} \triangleq\left[\bar{G}_{1}^{\mathrm{T}} \mid P_{1}\right], \quad U_{1}=\mathbf{1}_{l}^{l}, \quad V_{1}=\mathbf{1}_{N}^{N}, \quad N^{\mathrm{T}} N=\mathbf{1}_{n}^{n}$,

$$
R \triangleq\left[\bar{G}_{1}^{\mathrm{T}} \mid P_{1}\right]\left[\begin{array}{ll}
U & 0 \\
0 & V
\end{array}\right]\left[\frac{\bar{G}_{1}}{P_{1}^{\mathrm{T}}}\right] \triangleq N^{\mathrm{T}} W N,-W \triangleq\left[\begin{array}{ll}
U & 0 \\
0 & V
\end{array}\right]=(n \times n) .
$$

Равенство (3.1) выполнено лишь, если $\Phi_{A}^{-1} Q^{-1} \Phi_{\Omega}{ }^{-1} Q_{1}=R$. Отсюда по смыслу $\Phi_{\Omega}^{-1}$ необходимо и достаточно, чтобы $R$ удовлетворяла

$$
R \cdot=-\Psi^{-1}\left[\Omega^{\mathrm{T}} \Psi+\Psi \cdot\right] R, \quad \Psi \triangleq Q \Phi_{A}, . R_{1} \triangleq R\left[t_{1}, \cdot\right]=\mathbf{1}_{n}^{n} .
$$

Учитывая (3.2), из (3.3) находим

$W \cdot=M W, \quad M=\left[\begin{array}{ll}M_{11} & M_{12} \\ M_{21} & M_{22}\end{array}\right] \triangleq N \theta N^{\mathrm{T}}, \quad \theta \triangleq \Psi^{-1}\left[\left(\omega^{\mathrm{T}}-A^{\mathrm{T}}\right) \Psi-\Psi \cdot\right]$,

$M_{11}=\bar{G}_{1} \theta \bar{G}_{1}^{\mathrm{T}}, \quad M_{12}=\bar{G}_{1} \theta P_{1}, \quad M_{21}=P_{1}^{\mathrm{T}} \theta \bar{G}_{1}^{\mathrm{T}}, \quad M_{22}=P_{1}^{\mathrm{T}} \theta P_{1}, \quad \omega=B R^{-1} B^{\mathrm{T}} G^{\mathrm{T}} \bar{M} G$.

В силу (3.4) и структуры $W$ заключаем, что (3.1) верно лишь, если

$$
M_{12}(\theta)={ }_{l} 0^{N}, \quad M_{21}(\theta)={ }_{N} 0^{l} .
$$

Тогда уравнения (3.4) на $U, V$ совместны, разделяются и $U(t)=\Phi\left(M_{11}\right)$, $V(t)=\Phi\left(M_{22}\right), \Phi_{A} \triangleq \Phi(A)$. Но (3.5) верны лишь при равенстве $\theta=R \cdot R^{-1}$, эквивалентном ввиду $(3.2)-(3.4)$ параметрическому представлению множества допустимых (3.1) матриц $A, B, Q, R, \bar{A}, G_{1}$ уравнением

$$
\omega^{\mathrm{T}}=A^{\mathrm{T}}+(\Psi R) \cdot(\Psi R)^{-1},
$$

где используются формулы и обозначения $(2.15),(3.1)-(3.3)$. Связь (3.6) примет вид (3.7) в обозначениях (3.8)

$$
\hat{B} \hat{X}=\hat{C}+K \hat{Y} K^{-1} \quad \text { при } \quad r(B)=r(R)=r=l ;
$$

$$
\begin{gathered}
\hat{B}=\hat{B}^{\mathrm{T}} \triangleq B R^{-1} B^{\mathrm{T}}, \quad \hat{X} \triangleq G^{\mathrm{T}} \bar{M}(t, G, \cdot) G, \quad K \triangleq Q^{-1} \Phi_{A}^{-1 \mathrm{~T}} N^{\mathrm{T}}, \\
\hat{C}=A-Q^{-1}\left[Q \cdot A^{\mathrm{T}} Q\right], \quad N^{\mathrm{T}}\left(G_{1}\right)=\left[G_{1}^{\mathrm{T}}\left(G_{1} G_{1}^{\mathrm{T}}\right)^{-1 / 2} \mid P_{1}\right],
\end{gathered}
$$

$$
\hat{Y}^{\mathrm{T}}=\left[\begin{array}{cc}
U \cdot U^{-1} & 0 \\
0 & V \cdot V^{-1}
\end{array}\right], \quad \widetilde{B}=B \widetilde{T}, \quad \widetilde{R}=\widetilde{T}^{\mathrm{T}} R \widetilde{T}, \quad r(\widetilde{T})=l, \quad \widetilde{T}=(r \times l) .
$$

Ранговые равенства в (3.7) получим агрегацией $u=\widetilde{T} \tilde{u}$ в (3.8), coхраняя за $\widetilde{u}, \tilde{B}, \widetilde{R}$ исходные обозначения. Подставив в (3.7) его частное решение $\hat{X}=\hat{X}_{0}$ вида

$$
\hat{X}_{0}=B\left(B^{\mathrm{T}} B\right)^{-1} R\left(B^{\mathrm{T}} B\right)^{-1} B^{\mathrm{T}}\left(\hat{C}+K \hat{Y} K^{-1}\right) \quad\left(\Delta\left(B^{\mathrm{T}} B\right) \neq 0 ; r(B)=l\right)
$$

найдем условия совместности (3.7) 


$$
\left[B\left(B^{\mathrm{T}} B\right)^{-1} B^{\mathrm{T}}-\mathbf{1}_{n}^{n}\right]\left[\hat{C}+K \hat{Y} K^{-1}\right]=0_{n}^{n},
$$

эквивалентные независимым связям $\hat{R}\left[\hat{C}+K \hat{Y} K^{-1}\right]={ }_{N} 0^{n}$, где $(N \times n)$ матрица $\hat{R}(B)$ - любая, удовлетворяющая равенствам

$$
\hat{R} B={ }_{N} 0^{l}, \quad \hat{R} \hat{R}^{\mathrm{T}}=\mathbf{1}_{N}^{N}, \quad \hat{R}^{\mathrm{T}} \hat{R}=\mathbf{1}_{n}^{n}-B\left(B^{\mathrm{T}} B\right)^{-1} B^{\mathrm{T}} .
$$

Решая $(3.10),(3.11)$ относительно $\hat{C}$, приводим условия существования $\hat{X}$ в уравнении $(3.7)$ к $\hat{F}$ - параметрическому виду допустимых $A, B, Q$, $R$ с произвольной непрерывной $(l \times n)$-матрицей $\hat{F}(t)$

$$
\hat{C}=B \hat{F}+\left[B\left(B^{\mathrm{T}} B\right)^{-1} B^{\mathrm{T}}-\mathbf{1}_{n}^{n}\right] K \hat{Y} K^{-1}, \quad \partial \hat{F} / \partial G_{1}=0 .
$$

Здесь и ниже в силу (3.8) необходимо условие $\partial \hat{C} / \partial G_{1}=0$.

\section{Упрощенный вид условия декомпозиции (3.6) (субоптимальная сепарация)}

Достаточным и близким к необходимому простым условием выполнения (3.13) является равенство

$K \hat{Y} K^{-1}=\lambda \mathbf{1}_{n}^{n}+B \hat{V}\left(\right.$ где $\frac{\partial \lambda}{\partial G_{1}}=0$, а матрица $\hat{V}_{l}^{n}\left(t, G_{1}\right)-$ произвольная $)$,

эквивалентное $\hat{Y}=\lambda \mathbf{1}_{n}^{n}+K^{-1} B \hat{V} K$, где ${ }_{n} \hat{Y}^{n}-$ произвольная вида (3.8). Обозначим

${ }^{r} \hat{A}=l, \quad \hat{A}_{1}=(l \times l), \quad \hat{A}_{2}=(N \times l) ; \quad \hat{A}=\left[\begin{array}{c}\hat{A}_{1} \\ \hat{A_{2}}\end{array}\right] \triangleq K^{-1} B, \quad \tilde{V} \triangleq \hat{V} K$.

Согласно (3.8), (3.15) матрицы $\widetilde{\nabla}, \hat{Y}, \hat{C}$ в (3.12), (3.14) примут вид $\nabla=\left[\hat{H}_{1} W_{1} \mid \hat{H}_{2} \widehat{W}_{2}\right] ; \quad \hat{H}_{1}\left(l \times d_{1}\right): \hat{A}_{2} \hat{H}_{1}={ }_{N} 0^{d_{1}}, \quad d_{1} \triangleq l-r\left(\hat{A}_{2}\right) \geqslant 0$,

$\hat{H}_{2}\left(l \times d_{2}\right): \hat{A}_{1} \hat{H}_{2}={ }^{0} 0^{d_{2}}, \quad d_{2} \triangleq l-r\left(\hat{A}_{1}\right) \geqslant 0 ; \quad \mathscr{W}_{1}\left(d_{1} \times l\right), \quad \mathscr{W}_{2}\left(d_{2} \times N\right)-$ произвольны,

$\hat{Y}=\hat{Y}_{1} \triangleq \lambda \mathbf{1}_{n}^{n}+K^{-1} B \widetilde{V}, \hat{C}=\hat{C}\left(\hat{Y}_{1}\right) \triangleq \hat{C}_{1}=B \hat{F}+\lambda\left[B\left(B^{\mathrm{T}} B\right)^{-1} B^{\mathrm{T}}-\mathbf{1}_{n}^{n}\right] \quad(\lambda \geqslant 0)$, где $d_{1}+d_{2}=2 l-r\left(\hat{A}_{1}\right)-r\left(\hat{A}_{2}\right), \partial \lambda / \partial G_{1} \equiv 0 ; \lambda ; \hat{F}(l \times n)-$ произвольные. Заданная в $(3.8),(3.16)$ связь $A, Q$ удовлетворяется при преобразовании $x \rightarrow \widetilde{x}$. Из $(3.7),(3.16)$ в классе $(3.14)$ находим упрощенный вид допустимых $G\left(t, t_{1}, G_{1} \mid \bar{A}, \cdot\right)$ :

$$
G^{\mathrm{T}} \bar{M} G=B\left(B^{\mathrm{T}} B\right)^{-1} R\left[\lambda\left(B^{\mathrm{T}} B\right)^{-1}+\hat{F}+\hat{V}\right]+\hat{R}^{\mathrm{T}} \bar{N}^{\mathrm{T}} \bar{N} R \quad(r(\bar{N}) \leqslant l),
$$

где

$$
\hat{C}+K \hat{Y}_{1} K^{-1}=B\left[\hat{F}+\lambda\left(B^{\mathrm{T}} B\right)^{-1} B^{\mathrm{T}}+\hat{V}\right], \quad \lambda \geqslant 0, \quad \bar{N}=(N \times N)
$$


$\bar{N}=\bar{N}(t, \cdot)$ - произвольная матрица. Из симметрии $\hat{X}$ необходимо, чтобы

$$
B\left(B^{\mathrm{T}} B\right)^{-1} R[\hat{F}-\hat{V}]=[\hat{F}-\hat{V}] R\left(B^{\mathrm{T}} B\right)^{-1} B^{\mathrm{T}} .
$$

Условие (3.18) выполнено, если $\hat{F}, \widetilde{V}$ задать равенствами

$\hat{F}=\hat{\Psi} R\left(B^{\mathrm{T}} B\right)^{-1} B^{\mathrm{T}}, \quad 0 \leqslant \hat{\Psi}^{\mathrm{T}}=\hat{\Psi}(l \times l)-$ произвольная матрица,

$\widetilde{\nabla}=\hat{M} R\left(B^{\mathrm{T}} B\right)^{-1} B^{\mathrm{T}} K, \quad 0 \leqslant \hat{M}^{\mathrm{T}}=\hat{M}(l \times l)-$ совместная с (3.16) матрица.

В связи с (3.19) рассмотрим существование $\hat{M}_{1}(l \times l)$ в равенствах $\hat{M}_{1}=\hat{M}_{1}^{\mathrm{T}}, \quad \hat{M}_{1} S=\hat{H}_{1} \hat{W}_{1} \quad\left(S_{l}^{n}=R\left(B^{\mathrm{T}} B\right)^{-1} B^{\mathrm{T}}, r_{s}=l ; \hat{W}_{\gamma} \triangleq \mathscr{W}_{\gamma} K_{\gamma}^{-1}, \gamma=1,2\right)$.

Ввиду $\Delta\left(S S^{\mathrm{T}}\right) \neq 0$ существует единственное решение $(3.20)$ вида $\hat{M}_{1}$ в (3.21) при условии совместности (3.22), получаемого из них подстановкой

$$
\begin{gathered}
\hat{M}_{1}=\hat{H}_{1} \hat{W}_{1} S^{\mathrm{T}}\left(S S^{\mathrm{T}}\right)^{-1}, \\
\hat{H}_{1} \hat{W}_{1}\left[\mathbf{1}_{n}^{n}-S^{\mathrm{T}}\left(S S^{\mathrm{T}}\right)^{-1} S\right]=0_{l}^{n} .
\end{gathered}
$$

Отсюда необходимо, чтобы $\hat{H}_{1} \hat{W}_{1}=\hat{T}_{1} S$, где $\hat{T}_{1}(l \times l)-$ произвольна. Умножая (3.22) на $\hat{H}_{1}{ }^{\mathrm{T}}$ слева, получим $\hat{W}_{1}=\widetilde{M}_{1} S$, где $\widetilde{M}_{1}\left(d_{1} \times l\right)-$ произвольная. При этом необходимо и достаточно, чтобы $\hat{M}=\hat{H}_{1} \widetilde{M}_{1}$. С учетом симметрии и неотрицательности $\hat{M}_{1}$ примем $M_{1}=\tilde{\omega}_{1} \tilde{\omega}_{1}^{\mathrm{T}} \hat{H}_{1}^{\mathrm{T}}$, где $\tilde{\omega}_{1}=\left(d_{1} \times l\right)-$ произвольная матрица, $r\left(\tilde{\omega}_{1}\right)=d_{1} ; r\left(\hat{M}_{1}\right) \leqslant d_{1}$. Поэтому вопрос $\exists \hat{M}_{1}$ сводится к существованию $\mathscr{W}_{1}$ в равенстве

$$
\mathscr{W}_{1} K_{1}^{-1}=R_{1}=\tilde{\omega} \tilde{\omega}_{1}^{\mathrm{T}} \hat{H}_{1}^{\mathrm{T}} S \quad\left(\hat{W}_{1}=\widetilde{M}_{1} S=\widetilde{W}_{1} K_{1}^{-1}\right),
$$

где небходимо в силу $r\left(K_{1}^{-1}\right) \triangleq \operatorname{rank} K_{1}^{-1}=l$, чтобы

$$
W_{1}=R_{1} K_{1}^{-1}\left(K_{1}^{-1} K_{1}^{-1 \mathrm{~T}}\right)^{-1} \sim R_{1}\left[1_{n}^{n}-K_{1}^{-1 \mathrm{~T}}\left(K_{1}^{-1} K_{1}^{-1 \mathrm{~T}}\right) K_{1}^{-1}\right]=0_{d_{\perp}}^{n} .
$$

Считая $\tilde{\omega}_{1}$ свободной в $(3.23)$, необходимо условие принять

$$
\hat{H}_{1}^{-\tau} S=\hat{Q}_{1} K_{1}^{-1}, \text { где } \hat{Q}_{1}\left(d_{1} \times l\right)-\text { произвольная матрица. }
$$

При (3.25) условие (3.24) совместности (3.23) выполнено, причем

$$
\tilde{W}_{1}=\tilde{\omega}_{1} \tilde{\omega}_{1}^{\mathrm{T}} \hat{H}_{1}^{\mathrm{T}}, \quad \hat{M}_{1}=\hat{H}_{1} \tilde{\omega}_{1} \tilde{\omega}_{1}^{\mathrm{T}} \hat{H}_{1}^{\mathrm{T}} .
$$

Сменой индекса $1 \rightarrow 2$ аналогично $(3.20)-(3.26)$ находим условия существования $\hat{M}_{2}$ для $\hat{M}=\hat{M}_{1}+\hat{M}_{2}=(l \times l)$. Ниже в (3.16), (3.17) допустимы лишь параметры $\lambda, \hat{\Psi}, \bar{N}, \tilde{\omega}_{1}, \tilde{\omega}_{2}, \quad$ при которых ранг (3.17) есть $l$. Усиленные условия $z, y, \widetilde{M}, \widetilde{K}$ разделения (3.17), (3.25), (3.26) при $u=u_{\star}$ на $\bar{A}, G_{1}$ и параметры системы $(1.2)$ кратко назовем условиями субоптимальной сепарацин. 


\section{4. Выбор наилучшего субоптимального управления $u^{*}$}

Из (2.16), (2.17) имеем в $t$, $\xi$ субоптимум системы $(1.1),(1.2)$ в виде

$$
\tilde{S}^{*} \triangleq \tilde{f}\left(t, \xi \mid u^{*}\right)=1 / 2\left(z^{\mathrm{T}} \tilde{M} z+y^{\mathrm{T}} \widetilde{K} y\right) \quad(\bar{M}=\widetilde{M}(t)) .
$$

Переходя в (4.1) от $t$, $\xi$ к переменным $t, x$ получим

$$
\begin{gathered}
S^{*}=f\left(t, x \mid u^{*}\right)=1 / 2 x^{\mathrm{T}} L x ; \quad L=L_{1}^{\prime}+L_{2}^{\prime}, \quad L_{1}^{\prime}=G^{\mathrm{T}} \bar{M} G, \quad L_{2}^{\prime}=H^{\mathrm{T}} K H, \\
L_{11}^{\prime}=\alpha_{1}^{2} G_{1}^{\mathrm{T}} \widetilde{Q}_{11} G_{1}, \quad L_{21}^{\prime}=\alpha_{1}^{2}\left[Q_{1}-G_{1}^{\mathrm{T}} \widetilde{Q}_{11} G_{1}\right], \\
L=\left[\begin{array}{c}
G \\
H
\end{array}\right]^{\mathrm{T}}\left[\begin{array}{cc}
\bar{M} & 0 \\
0 & \widetilde{K}
\end{array}\right]\left[\begin{array}{c}
G \\
H
\end{array}\right] \quad\left(L^{\prime}{ }_{\gamma 1}=L_{\gamma}^{\prime}\left(t_{1}\right), \gamma=1,2\right) .
\end{gathered}
$$

Так как $L, L_{1}^{\prime}$ не зависят от свободных параметров $\overline{\bar{A}}, W_{1}{ }^{0}$ матрицы $H$ в $(2.16),(3.1)$, то геометрически очевидна независимость от них $L_{2}{ }_{2}$. Действительно, из $(2.2),(2.10),(4.2),(2.16)$ выводим равенство

$$
L_{2}^{\prime}=\Phi_{\Omega}^{-1 \mathrm{~T}}[t]\left\{L_{21}^{\prime}-\int_{t}^{t_{\mathrm{r}}} \Phi_{\Omega}^{\mathrm{T}}\left[Q-G^{\mathrm{T}}\left(G Q^{-1} G^{\mathrm{T}}\right)^{-1} G\right] \Phi_{\Omega} d \tau\right\} \Phi_{\Omega}^{-1}[t],
$$

доказывающее утверждение. Для $L_{1}$ с учетом $(2.15),(2.18)$ получаем

$$
\begin{gathered}
-L_{1}^{\prime}=\Omega^{\mathrm{T}} L_{1}^{\prime}+L_{1}^{\prime} \Omega+G^{\mathrm{T}} \hat{Q}_{1} G \\
\left(\hat{Q}_{1}=\widetilde{Q}_{1}+\bar{M} G B R^{-1} B^{\mathrm{T}} G^{\mathrm{T}} \bar{M}, \widetilde{Q}_{1}=\left(G Q^{-1} G^{\mathrm{T}}\right)^{-1}\right), \\
L_{1}^{\prime}=\Phi_{\Omega}^{-1 \mathrm{~T}}[t]\left\{L_{11}^{\prime}-\int_{t}^{t_{1}} \Phi_{\Omega}^{\mathrm{T}} G^{\mathrm{T}} \hat{Q}_{1} G \Phi_{\Omega} d \tau\right\} \Phi_{\Omega}^{-1}[t],+\Omega \triangleq-B R^{-1} \bar{B}^{\mathrm{T}} \bar{M} G+A .
\end{gathered}
$$

Сложением $L_{2}^{\prime}, L_{1}^{\prime}$ в (4.3), (4.4) находим функционал от $\bar{A}, G_{1}$

$$
\begin{gathered}
L\left(t, t_{1} \mid \bar{A}, G_{1}\right)= \\
=\Phi_{\Omega}^{-1 \mathrm{~T}}(t)\left\{\alpha_{1}^{2} Q_{1}+\int_{t}^{t_{1}} \Phi_{\Omega}^{\mathrm{T}}\left[Q+G^{\mathrm{T}} \bar{M} G B R^{-1} B^{\mathrm{T}} G^{\mathrm{T}} \bar{M} G\right] \Phi_{\Omega} d \tau\right\} \Phi_{\Omega}^{-1}(t) \\
L_{11}^{\prime} \stackrel{\Delta}{=} L_{1}^{\prime}\left(t_{1} ; t_{1}, \cdot\right)=\alpha_{1}^{2} \widetilde{Q}_{11}, . L_{1} \triangleq L\left(t_{1}, t_{1}, \cdot\right)=\alpha_{1}^{2} Q_{1} .
\end{gathered}
$$

Выбор наилучшего субоптимального регулятора (2.7) подчиним задаче минимизации меры $I$-невязки (4.5) в $t=t_{0}$ вероятностного типа [ $\left.^{3,5}\right]$ при условиях (3.17), (3.25), (3.26). В виде меры $I$-невязки субоптимальности, минимизируемой на параметрах $\bar{A}, G_{1}, \hat{\Psi}, \bar{N}, \tilde{\omega}, \tilde{\omega_{2}}$, трактуемых как управления, возьмем симметрические полиномы от собственных чисел матрицы (4.5) в $t=t_{0}, \quad$ в частности, $I_{1}=\operatorname{tr} L\left(t_{0}, \cdot\right), I_{n}=\operatorname{det} L\left(t_{0}, \cdot\right)$. Используя матричные множители Лагранжа системы (1.2), (2.8), (4.5) с учетом условий сепарации, на основе анализа первой вариации $I$ получим аналитический вид необходимых условий выбора наилучших матриц $\bar{A}^{0}, G_{1}{ }^{0}\left(V^{0}=V_{\text {opt }}\right)$ аналогично процедурам $\left[{ }^{3-5,7}\right]$. В практическом поиске $I$-оптимальных $V^{0}$ предпочтительнее численные методы (напр., метод градиентов) [ $\left.{ }^{4,6}\right]$. В ряде случаев (напр., при ортогональных преобразованиях $T$ в (2.1), либо при инвариантных по $T$ мерах $I$ ) объем вычислений сокращается в переменных $x, y, \tilde{L}$ в силу блочного вида (4.1) и разделения системы (2.16). 


\section{ЛИТЕ РА Т У}

1. Aoki, M. Joint Automat. Contr. Conf. Preprints Papers, New York, 178-183 (1967).

2. Aoki, M. IEEE Trans. Automat. Contr., 13, 246-248 (1968).

3. Kleinman, D. L., Athans, M. IEEE Trans. Automat. Contr., 13, 150-153 (1968).

4. Ульм С. Изв. АН ЭССР. Физ. Матем., 19, № 2, 150-151 (1970).

5. Ульм С. Изв. АН ЭССР. Физ. Матем., 20, № 1, 3-7 (1971).

6. Васильев Ф. П. Численные методы решения экстремальных задач. М., «Наука», 1980.

7. Болтянский В. Г. Математические методы оптимального управления. М., «Наука», 1966.
Институт кибернетики
Академии наук Эстонской ССР
Поступнла в редакцию
23/IX 1983

I. KEIS

\section{LINEAARNE MITTESTATSIONAARNE AGREGEERIMISMEETOD SUBOPTIMAALSETE JUHTIMISTE SUNTEESIMISEKS}

On vaadeldud ruutkriteeriumiga lineaarse mitmemõõtmelise dünaamilise seotud tingimustega (1.7), (2.15) süsteemi (1.1), (1.2) mittestatsionaarset $z$-agregeerimist koordinaatide järgi (1.5), (2.1), mis vōimaldab üldistada Aoki-Athans'i konstantset agregeerimismaatriksit kasutava meetodi. On leitud Liouville'i valemi üldistuseks modifikatsioon (1.18) ning saadud ülesande suboptimaalsed regulaatorid (2.7) ja hinnangud (2.21) $z$-normi määramiseks reguleerimisajavahemikus.

\section{KEIS}

\section{ON THE LINEAR UNSTATIONARY AGGREGATION IN THE SUBOPTIMAL CONTROL SYNTHESIS}

The paper deals with the control problem of the linear multidimensional $(n \gg 2)$ dynamical system (1.2) governed by the quadratic performance criterion of quality. The aggregation viewpoint, aiming to diminish the computational procedures volume, is taken into account.

A new approach is introduced on the basis of unstationary linear transform (1.5), (2.1), defined by time-dependent aggregation matrixes, instead of the constant ones in Aoki-Athans way. The principal scheme includes three following stages.

1. The introduction of the $z$-aggregative component (1.5) composed of informative variables (1.3) and its $y$-complementing component (2.1), satisfying separation condition (1.7), (2.15), respectively. Here the modification (1.18) of Liouville formula is obtained.

2. The aggregation in performance index (2.4) relevant for orthogonality condition (2.2), is provided in (2.6).

3 . The optimal aggregation matrix choice for the prescribed criterion under exact conditions (3.6) or their simplified form (3.17), (3.25), (3.26), is investigated.

As a result, suboptimal control policy is derived in $(2.7)$, and the evaluations (2.21) for $z$-norm during the time of regulation are obtained. 